\title{
Assessment of serum copper, iron and immune complexes in potentially malignant disorders and oral cancer
}

\section{Ritu TIWARI(a) \\ Chaya Manoranjini DAVID(a) \\ Datturao Ramanand MAHESH ${ }^{(a)}$ \\ Uma SAMBARGI(a) \\ Kademadkal Javaraiah RASHMI(a) \\ Prabhavati BENAKANAL(a)}

(a) DayanandaSagar College of Dental Sciences, Department of Oral Medicine and Radiology, Karnataka, India

Declaration of Interests: The authors certify that they have no commercial or associative interest that represents a conflict of interest in connection with the manuscript.

Corresponding Author:

Ritu Tiwari

E-mail: tiwari.ritu28@gmail.com

DOI: 10.1590/1807-3107BOR-2016.vol30.0101

Submitted: Dec 09, 2015

Accepted for publication: Apr 25, 2016

Last revision: June 18, 2016
Abstract: Potentially malignant disorders (PMDs) of oral cavity and oral cancer remain a cause of serious concern despite intensive research and development. Diet and immunity have been identified to play a crucial role as modifying factors in these diseases. Our study intended to explore this relationship by estimating and comparing the serum levels of copper, iron and circulating immune complexes (CICs) in patients diagnosed with PMDs and oral cancer and normal healthy individuals. In this study, 40 histopathologically diagnosed cases of PMDs and oral cancer were included along with 30 healthy controls and $5 \mathrm{ml}$ of venous blood was drawn using venipuncture. Serum estimation of copper, iron and CIC then followed using the colorimetric and spectrophotometric methods. The data obtained was subjected to statistical analysis using one way ANOVA and Pearson's Product-Moment Correlation Test. The mean serum copper level was measured as $138.98 \pm 10.13 \mu \mathrm{g} / 100 \mathrm{ml}$ in the PMD group and $141.99 \pm 21.44 \mu \mathrm{g} / 100 \mathrm{ml}$ in the oral cancer as compared to $105.5+18.81 \mu / 100 \mathrm{ml}$ in the controls. The mean serum CIC levels was highest in the oral cancer $\left(9.65 \pm 0.16 \mathrm{OD}_{470}\right)$ followed by the PMD group $\left(0.18+0.21 \mathrm{OD}_{470}\right)$ and least in the control group $\left(0.048 \pm 0.02 \mathrm{OD}_{470}\right)$. Whereas, the serum levels of iron showed a significant decrease in the PMD group $(110.9 \pm 10.54 \mu \mathrm{g} / 100 \mathrm{ml})$ and the oral cancer group (114.29 $\pm 25.83 \mu \mathrm{g} / 100 \mathrm{ml})$ as compared with the control group $(136.85 \pm 14.48 \mu \mathrm{g} / 100 \mathrm{ml})$. There was no positive correlation obtained between the three groups with respect to the chosen parameters indicating that the variables were independent of each other. It can be thus be ascertained that trace elements like copper and iron as well as humoral responses (CICs) have a close relationship with PMDs and oral cancers.

Keywords: Copper; Antigen-Antibody Complex; Iron; Mouth Neoplasms; Leukoplakia, Oral.

\section{Introduction}

Oral cancer is on the rise worldwide and is regarded as the sixth most common type of cancer. From a clinicopathological perspective, carcinomas can be divided into three categories: carcinomas of the oral cavity proper, carcinomas of the lip vermilion and carcinomas arising in

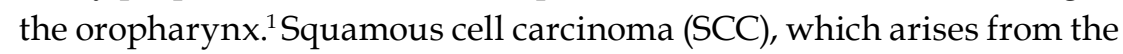
oral mucosal lining, accounts for over 90 percent of these tumors. ${ }^{1}$ These 
cancers are characterized by marked geographic, racial and gender disparities. The worldwide annual incidence of oral cancer is estimated to be 274,300 cases with 128,000 deaths. ${ }^{2}$ A great majority of new cases (more than 60\%) are from the developing world including India. ${ }^{3}$ India, being a major contributor to population explosion is likely to run a great projected risk of increasing the cancer burden to $75 \%$ by 2020 . $^{3}$

The mouth and pharynx which are directly exposed to both inhaled and ingestible carcinogens like tobacco are at a higher risk for showing malignant changes. Due to its association with these habits, oral cancer is more common in males but is increasingly being seen in females as well. ${ }^{2,3}$ Eighty-one percent of patients with Oral Squamous Cell Carcinomas (OSCCs) survive for at least one year following diagnosis, while the five-year relative survival rate for all stages of OSCCs is approximately $50 \%{ }^{1}$

There exists a definite need to expedite the process of diagnosis and treatment of oral cancers. Also, prompt screening and diagnosis of PMDs has an important role to play as they have a significantly high rate of malignant transformations. The prevalence of PMDs is much more than that of OSCC and they provide useful clinical markers for oral cancer. A major limitation however, is posed by inadequate means to differentiate PMDs at greater risk of progressing into invasive carcinoma from those at lower risk.

The development of PMDs and oral cancer is a multistep process with several secondary modifying factors like diet and immunity. ${ }^{3}$ Micronutrients like copper and iron are integral dietary components and have been the subject of recent studies focused on eliciting their role in pathogenesis and progression of these lesions. Since trace elements are necessary for cellular metabolism, any alteration in their level is of diagnostic significance as it will consequently affect the cell physiology., In addition, altered immunological status and immune system dysfunction has also been implicated in etiopathogenesis of malignancy. ${ }^{6}$ Research in early cancer detection has led to discovery of many immunological markers like circulating immune complex ( CIC).A strong co-relation exists between their levels and progression to oral cancer and to a slightly lesser extent in oral pre-malignancy.
Since prognosis rests heavily on early diagnosis, there is a need to establish biomarkers which can promptly and reliably detect the presence of the disease. Keeping in view the aforesaid facts, present study was designed to estimate the serum levels of copper, iron and CICs in patients diagnosed with PMDs, oral squamous cell carcinoma and healthy individuals, Also, the three parameters were subjected to comparative co-relation test to determine the variation in their levels with respect to each other.

\section{Methodology}

The study was approved by the research and ethics committee of the institute. A total of 110 patients, aged 25-70 years, were chosen from the outpatient Department of Oral Medicine and Radiology, Dayananda Sagar College of Dental Sciences Bangalore, Karnataka and Oncology departments of various hospitals in Bangalore. The exclusion criteria were patients with known allergies, autoimmune diseases (except oral lichen planus), diabetes mellitus, hypertension, myocardial infarction, acute infections, acute or chronic liver disease, nephrotic syndrome or previous history of treatment for cancers (surgery, chemotherapy, radiotherapy). Such patients were excluded on the basis of an extensive medical history questionnaire, following which written informed consent was taken from the healthy individuals before being considered for the study.

The subjects were segregated into three groups; 30 healthy individuals were taken as controls forming the Group I, 40 clinically diagnosed and histopathologically proven cases (moderate and severe dysplasia) of leukoplakia (14), erosive oral lichen planus (EOLP) (7) and clinically diagnosed oral submucous fibrosis(OSMF) (19) were considered in the group of PMDs (Group II) and third group was clinically diagnosed and histopathologically proven 40 cases of oral squamous cell carcinoma (OSCC).

Blood was chosen as the investigative medium, although it being invasive in nature, the procedure for blood collection is standardized, cost-effective and easily reproducible as compared to that of fluids like saliva. Also, cross-contamination and stability of standards and controls are more precisely attainable with blood/serum samples. 
Venous blood samples were collected from the mid-cubital vein in $5 \mathrm{ml}$ disposable syringe using aseptic precautions. It was then transferred to a sterile glass tube and allowed to clot for $60 \mathrm{~min}$ followed by centrifugation at 2,000 rpm for 10 minutes. Serum thus separated was subjected to the following tests:

Serum levels of Copper $(\mathrm{Cu})$ were determined using the Colorimetric Di-Br-PAESA method (Coral Copper Estimation Kit, Tulip Group India) with semi-automated analyzer (Erba Mannheim' Chem-7 Semiautomated Analyzer). Serum analysis of Iron $(\mathrm{Fe})$ was done using Colorimteric Ferrozine method with semi-automated analyzer (Coral Copper Estimation Kit, Tulip Group India).Serum CIC was determined using 3.75\% Polyethylene Glycol (PEG) serum precipitation method by Prabha Balram (1987) using spectrophotometry (Uv-1700 Pharmaspec Uv-Vis Spectrophotometer, Shimadzu).

Statistical analysis of the data obtained was done using one way ANOVA test and the Pearson's Product-Moment Correlation Test. In all the above tests a p-value of less than 0.001 was accepted as indicating high level of statistical significance.

\section{Results}

Group wise comparison of gender among the cases was performed. In the PMD group (leukoplakia/EOLP/OSMF), females were 25\% (10) and males formed $75 \%$ (30) of the subjects. In the oral cancer group, females attributed to $40 \%$ (16) and males to $60 \%$ (24) of the subjects. In the control group, $56.7 \%$ (17) were males and $43.3 \%$ (13) were females (Figure 1). The mean age (in years) of the patients with potentially malignant disorder was $41.1 \pm 12.14$ in the PMD group as compared to $55.52 \pm 12.10$ in oral cancer group and $33.26 \pm 7.72$ in the control group. The mean age in PMDs and oral cancer group was higher than the controls (Figure 2).

The mean serum copper levels were found to be $138.98 \pm 10.13 \mu \mathrm{g} / 100 \mathrm{ml}$ in the PMD group, $141.99 \pm 21.44 \mu \mathrm{g} / 100 \mathrm{ml}$ in the oral cancer group and $105.5 \pm 18.81 \mu / 100 \mathrm{ml}$ in the control group. Highly significant difference $(p<0.001)$ between the three groups were obtained, with lowest levels being in control group (Figure 3, Table 1).
The mean serum iron levels were found to be $110.9 \pm 10.54 \mu \mathrm{g} / 100 \mathrm{ml}$ in the PMD group, $114.29 \pm 25.83 \mu \mathrm{g} / 100 \mathrm{ml}$ in the oral cancer group and $136.85 \pm 14.48 \mu \mathrm{g} / 100 \mathrm{ml}$ in the control group. The

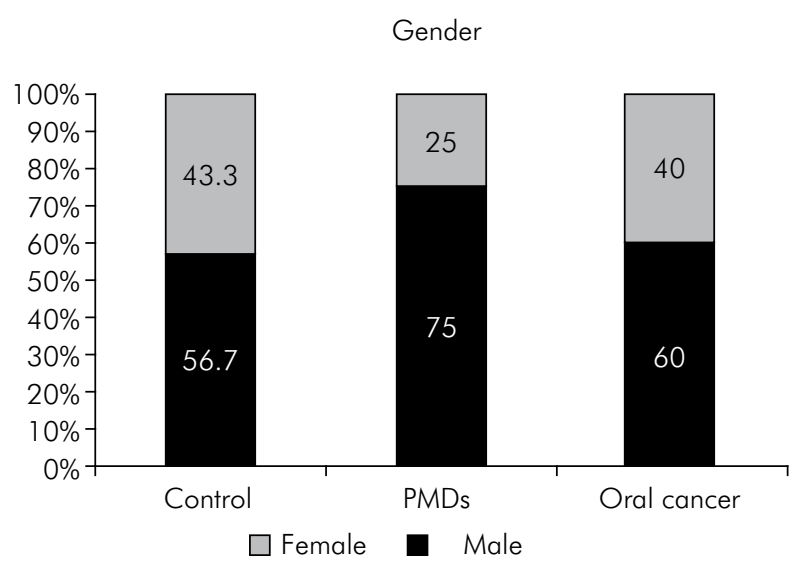

Figure 1. Bar Graph showing distribution of gender among the three groups.

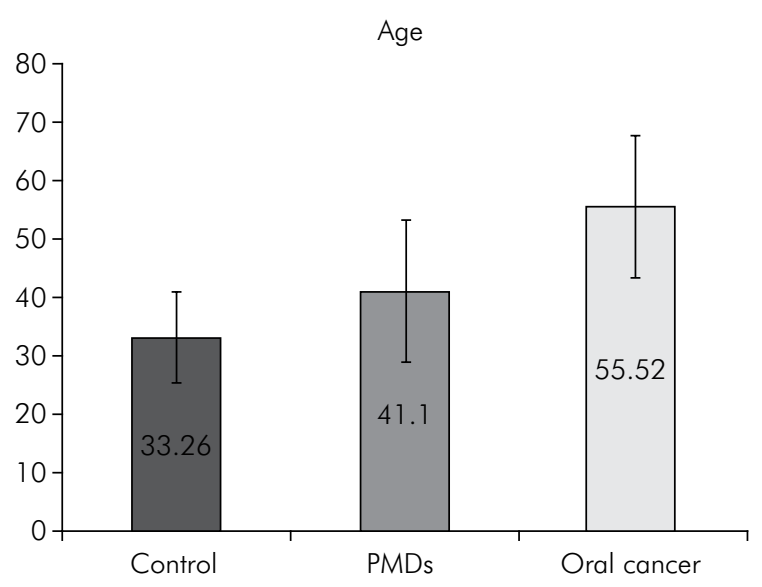

Figure 2. Bar Graph showing distribution of age among the three groups.

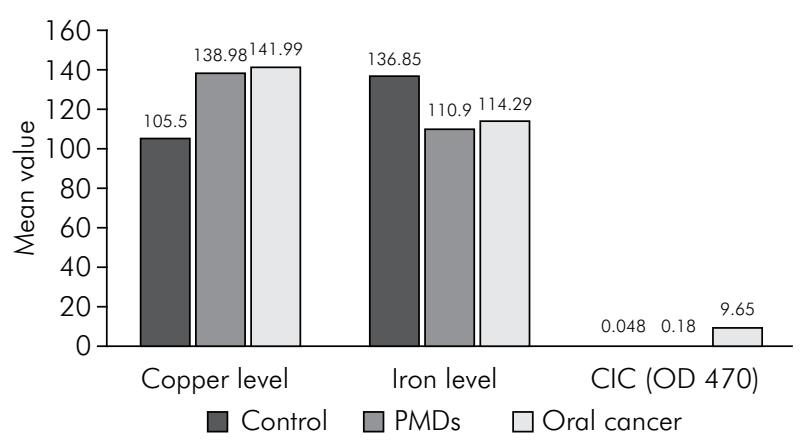

Figure 3. Groupwise comparison of serum copper, iron and $\mathrm{CIC}$. 
difference between the three groups was found to be highly statistically significant with $p$ value $<0.001$, maximum being in the control group (Figure 3, Table 1).

The mean serum CIC levels were found to be $0.18 \pm 0.21 \mathrm{OD}_{470}$ in the PMD group, $9.65 \pm 0.16 \mathrm{OD}_{470}$ in the oral cancer group and $0.048 \pm 0.02 \mathrm{OD}_{470}$ in the control group. The mean serum CIC values were elevated in the study group (PMD and oral cancer group) when compared with the normal individuals. There was a marked increase in the serum CIC levels in the oral cancer group as opposed to the PMD group. The obtained $p$ value for all the three groups were found to be $<0.001$ showing highly significant variation in the CIC levels from the control group through PMDs, to the oral cancer group (Figure 3, Table 1).

It was observed that the mean serum copper levels in leukoplakia, EOLP and OSMF patients progressively increased depending on the grade of dysplasia and clinical staging of the disease Also, mean serum iron levels in EOLP patients with severe dysplasia were comparatively less than patient with moderate dysplasia. No such observations were made with respect to the CIC levels in the three subgroups of PMDs (Table 2). No correlation was observed between the serum CIC levels and copper levels in the PMD group $(\mathrm{R}=0.088)$ or oral cancer group $(R=0.14)$ (Figure 4 and 5). Negative correlation was seen between the serum iron and copper values in the PMD $(R=-0.12)$ and oral cancer $(R=-0.071)$. Negative correlation was also obtained between the serum iron and CIC levels in the PMD group $(\mathrm{R}=-0.158)$ and in oral cancer $(\mathrm{R}=-0.108)$.

\section{Discussion}

Factually the death rate associated with oral cancer is quite high not because it is hard to discover or diagnose, but due to the reason that cancer is routinely discovered at an advanced stage. ${ }^{7}$ In its early stages it often goes unnoticed by the patient as it prospers without producing many symptoms. Late stage detection still remains a common occurrence owing to the dearth of a comprehensive program to resourcefully screen for the disease.

Oropharyngeal cancer has an associated high risk of producing second primary tumors (SPTs) which has been defined as new lesions that can arise either from the same genetically altered "field" as the primary tumor or independently from a different clone. ${ }^{8}$ Often a patient who survive the first encounter with the disease has up to 20 times higher risk of

Table 1. Groupwise comparison of variables (mean + SD)

\begin{tabular}{|c|c|c|c|c|c|}
\hline Variables & Control $(n=30)$ & PMDs $(n=40)$ & Oral cancer $(n=40)$ & F value & $p$ value \\
\hline Copper level & $105.5+18.81$ & $138.98+10.13$ & $141.99+21.44$ & 44.52 & $<0.001^{*}$ \\
\hline Iron level & $136.85+14.48$ & $110.9+10.54$ & $114.29+25.83$ & 19.1 & $<0.001^{*}$ \\
\hline $\mathrm{CIC}\left(\mathrm{OD}_{470}\right)$ & $0.048+0.02$ & $0.18+0.21$ & $9.65+0.16$ & 45279 & $<0.001^{*}$ \\
\hline
\end{tabular}

${ }^{*} p<0.001$ highly significant; ANOVA

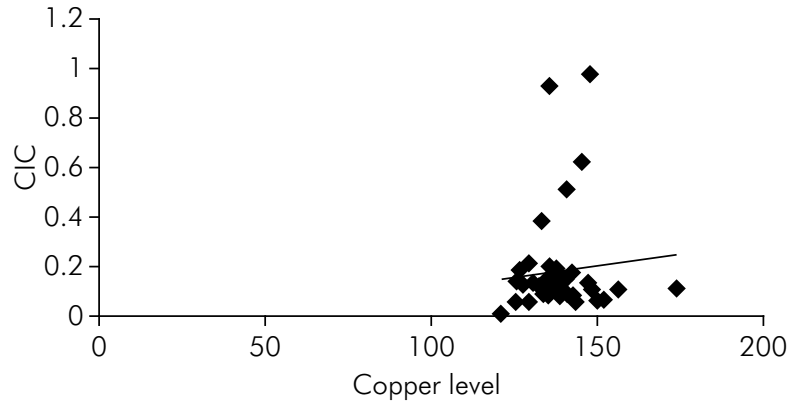

Figure 4. Correlation between $\mathrm{ClC}$ and Copper in the PMD group $(R=0.088)$.

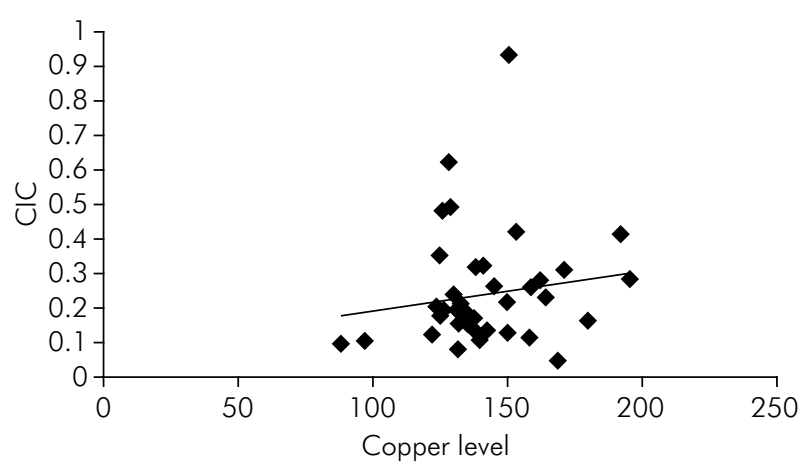

Figure 5. Correlation between $\mathrm{CIC}$ and copper in the oral cancer group $(R=0.14)$. 
Table 2. Mean serum copper, iron and CIC levels for PMD group (Homogenous leukoplakia, EOLP and OSMF) depending on grade of dysplastic changes.

\begin{tabular}{|c|c|c|c|c|c|}
\hline \multirow{2}{*}{ PMD } & \multirow{2}{*}{ Dysplasia } & \multirow{2}{*}{ No. of patients } & Mean Serum Copper & Mean Serum Iron & Mean $\mathrm{CIC}$ \\
\hline & & & $(\mu \mathrm{g} / \mathrm{dl})$ & $(\mu \mathrm{g} / \mathrm{dl})$ & $\left(O D_{470}\right)$ \\
\hline \multirow{2}{*}{ Homogenous leukoplakia } & Moderate & 10 & $133.18 \pm 5.05$ & $108.74 \pm 7.22$ & $0.239 \pm 0.25$ \\
\hline & Severe & 4 & $147.14 \pm 6.53$ & $115.83 \pm 15.10$ & $0.096 \pm 0.034$ \\
\hline \multirow{2}{*}{ EOLP } & Moderate & 6 & $138.69 \pm 12.69$ & $108.64 \pm 13.68$ & $0.307 \pm 0.37$ \\
\hline & Severe & 1 & 174.2 & 103.1 & 0.113 \\
\hline \multirow{4}{*}{ OSMF } & \multicolumn{5}{|c|}{ Clinical Staging (Pindborg's) } \\
\hline & Stage I & 13 & $134.60 \pm 4.76$ & $112.26 \pm 9.44$ & $0.119 \pm 0.04$ \\
\hline & Stage II & 5 & $146.50 \pm 4.02$ & $116.31 \pm 9.03$ & $0.193 \pm 0.23$ \\
\hline & Stage III & 1 & 152.34 & 120.63 & 0.065 \\
\hline
\end{tabular}

recurrence. $^{7}$ This is because carcinogenesis involves a multistep process which includes accumulation of multiple genetic and epigenetic shifts. Multifocal areas of cancers and PMDs develop from various genetically distinct clones and lateral spread of genetically related pre-invasive clones. ${ }^{9}$ This concept of field cancerization was proposed by Slaughter et al according to which several cell lines undergo neoplastic transformation independently, under the stress of regional carcinogenic activity. ${ }^{10}$ Local tumor recurrence following complete surgical resection also credits the existence of morphologically intact and genetically damaged cells. ${ }^{11}$ Novel molecular studies have established that a critical genetic alteration in a single cell can confer a growth advantage over the neighboring cells. The cells harboring faulty genes then tend to migrate towards adjacent mucosae and can accumulate other mutations eventually transforming them into aggressive disjointed sub clones phenotypically presenting as SPTs. ${ }^{11}$

Damage to genes may be due to several internal or external factors. There are studies indicating that diet low in nutrients could be a risk factor whereas, one high in these may have a protective value against many types of cancers. ${ }^{7}$ Impaired cell-mediated immunity has also been reported in patients with OSCCs. ${ }^{12}$ The major deviations in immune parameters in such patients appear to be increased leukocyte and lymphocyte counts, increased B- and T-cells and increased levels of Circulating Immune Complexes (CICs). ${ }^{13}$ Assuming that the involved antigens are malignancy-specific, serum CIC levels in cancer patients can be used for early diagnosis, assessment of metastatic spread, tumor burden, degree of aggressiveness, therapeutic response as well as prognosis. ${ }^{6}$

Age greater than 45 years is one among the established risk factors for oral cancer., ${ }^{1,12}$ It was found that more than $90 \%$ of OSCCs occur among patients older than 40 years of age. ${ }^{12}$ Average age of population affected with PMDs is 50-69 years, considered to be about 5 years earlier than oral cancer. PMDs have typically shown a predilection for males due to the presence of adverse habits. But recent research suggests a 1:1 male to female ratio. Our study group largely consisted of males who had habits of smoking and chewing tobacco or areca nut and PMDs were found to have a male predilection. Coelho et al had established that age-adjusted rates of oral cancer in India is high, being 20 per 100,000 population. ${ }^{14}$ In this study, $60 \%$ of patients detected with oral cancer were males and $40 \%$ were females and the control group comprised of $56.7 \%$ males and $43.3 \%$ females. Hence, the mean age of occurrence for OSCC and the PMDs was in accordance with previously reported data.

The control group of healthy individuals had a mean age of $33.26 \pm 7.72$ years that is less than the mean age of occurrence of OSCC but recent data has shown increased incidence of PMDs and OSCC in patients less than 40 years of age as well. ${ }^{15}$ Also, it has been observed that serum levels of micronutrients like copper and iron alters with increasing age. Madracic et al. had established that serum copper concentrations correlated positively with age $(p<0.0001)$. In elderly subjects 
above 75 years, a marked increase in serum copper concentrations were observed. ${ }^{16}$ Similarly, a study by Fairweather SJ et al. showed that iron deficiency anemia was prevalent in subjects above 80 years of age. This could be attributed to chronic low-grade inflammation that interferes with iron absorption and its metabolism eventually leading to depleted iron stores. ${ }^{17}$ Considering covariant like age and sex which have an important bearing on levels of trace elements, patients in the age range of 25-70 years were chosen as controls in the study to compare against the study group of PMDs and oral cancer.

In the present study, serum levels of copper were evaluated which showed a gradual increase from PMD to oral cancer as compared to the control group. Copper is present in many enzymes involved in oxidation (Tyrosinase, ceruloplasmin, amine oxidase, cytochrome oxidase) and is needed for maintaining cellular proton homeostasis. In PMDs there is an increased oxidative stress that can explain the increased levels of copper to combat the formation of reactive oxygen species (ROS).

OSMF has been described by Rajendran et al. as an altered oral mucosa subsequent to chronic deficiency of iron and/or vitamin "B" complex especially folic acid. Local irritants cause additional hypersensitivity and result in persistent juxtaepithelial inflammation, an initiating factor leading to a defective inflammatory-reparative response, culminating in fibrotic healing. ${ }^{18}$ OSMF is associated with habit of chewing areca nut and the copper from areca nut is known to increase collagen production in oral fibroblasts by up regulating lysyl oxidase leading to cross linking of collagen and elastin. ${ }^{19}$ In the present study as well, the OSMF patients were habitual areca nut chewers that can be held responsible for elevated serum copper levels. Increased serum copper in OSMF patients was also concomitant with the findings of elevated tissue and salivary copper levels in OSMF. ${ }^{5}$

The increased level of copper in OSCC is possibly due to an increase in cuproenzyme, ceruloplasmin and decreased catabolism of copper. ${ }^{19}$ Copper metabolism is profoundly altered in neoplastic disease, concurrently serum copper correlates with the tumor incidence and burden, its progression and recurrence in a variety of human cancers. Copper activates several angiogenic factors (VEGF, TNF-a, IL -1, b-FGF) which bind to endothelial cells, propelling them to enter G1 phase from G0 phase thus causing proliferation. ${ }^{5}$ Also, levels of ceruloplasmin, the principal copper transporting protein, increases four to eight folds during malignant progression. ${ }^{19}$

Another trace element, iron was estimated in the serum of the patients in this study. A statistically significant reduction was found in the PMD group and oral cancer group. The lowered level of iron appears to be the effect of the disease process rather than its cause. Serum iron levels are considered biochemical indicators for nutritional assessment. The cancer cells also have essentially the same qualitative iron requirements like the normal cells. They express their own transferrin receptors to obtain iron which may explain depletion of iron in such states. Hence, hypoferremia can be seen in association with carcinogenesis and iron levels can serve as prognostic indicator. Iron overload on the other hand has been known to aid carcinogenesis by provoking DNA damage. Iron induced oxidative stress causes redox regulation failure leading to lipid peroxidation and DNA and protein damage. Iron binding sites on macromolecules serve as centers for repeated production of hydroxyl radicals generated via the Fenton reaction. ${ }^{20} \mathrm{It}$ is considered that iron and oxygen together constitute a biologically damaging mixture due to increased formation of free radicals. ${ }^{20,21}$

Naturally occurring protein-bound iron, such as ferritin and transferrin, which constitute major forms of transport and storage of iron in vivo, produce considerable mitigation of free radical mediated toxicity in vivo and iron deficiency reduces this protection..$^{20}$ Shetty SR et al. observed that salivary iron was decreased in OSMF, OL and OSCC patients. ${ }^{22}$ Sethuraman $\mathrm{R}$ showed that serum iron levels were decreased in PMDs and oral cancerous groups when compared with the control group. ${ }^{23}$

Utilization of iron in collagen synthesis by hydroxylation of proline and lysine leads to decreased serum iron levels in OSMF patients. In most cases clinical anemia may also be a contributing factor. ${ }^{24}$ Karthik H et al..$^{25}$ estimated the hemoglobin and serum iron levels among healthy subjects and patients with OSMF in which latter showed significantly lower 
levels of hemoglobin and serum iron. Studies on diet and cancer have also suggested an increased risk of oral precancerous lesions among those women in the lowest quartile of iron intake (an increase of approximately 2.5 -fold) compared with other women. ${ }^{21}$

Serum iron and copper levels are likely to change due to pregnancy, estrogen therapy, systemic illness like myocardial infarction, hypertension, diabetes mellitus, thyrotoxicosis, liver cirrhosis, alcohol consumption. ${ }^{19}$ As these possible factors except alcohol (as it is one of the etiologic agents in cancer development) were excluded from this study, altered levels can be correlated with the potentially malignant and malignant states.

In this study, levels of CIC showed a gradual increase in the PMD group and oral cancer group. CICs have been implicated in autoimmune diseases, neoplastic diseases, infections caused by bacteria, viruses and parasites. ${ }^{6}$ They bind to inflammatory cells and stimulate local secretion of cytokines and vasoactive mediators which cause increased adhesion of leukocytes to the endothelium, vascular permeability and enhanced deposition of immune complexes in vessel walls by enlarging inter-endothelial spaces. This may further lead to amplification of tissue injury and disease. It also leads to suppression of cell mediated immunity and modulates the humoral response. ${ }^{6}$

CICs were first suggested as a useful tumor marker system for colorectal cancer by Chester $\mathrm{K}$ et al. ${ }^{26}$ Saranath DS et al. further evaluated the immune status in patients with oral cancer and found that CICs were elevated in all the untreated cases. ${ }^{27}$ In a study by Parveen $S$ et al. the immunoglobulin and CIC levels were estimated in the pretreatment and post treatment phases of oral cancer. Significantly elevated levels were found in the study group indicative of tumor burden which showed a comparative decline when compared with the post treatment phase. ${ }^{28}$ Rai S et al. observed that the mean CIC level in the control group was significantly lower when compared with the PMD group and malignant group. Among the potentially malignant group, leukoplakia had the highest mean CIC level followed by oral lichen planus and OSMF, suggesting a greater malignant potential associated with leukoplakia. ${ }^{29}$ Jane $\mathrm{C}$ et al investigated CICs in serum samples of 60 oral cancer patients having different grades of the disease and 40 patients with PMDs. Progressively elevated levels were observed in advanced stages of oral cancer patients and patients with PMDs. ${ }^{30}$ From these results it can be concluded that CIC represent the host's physiological and immunological defense response in eliciting specific antibodies upon exposure to most antigenic substances.

In this study, no statistically significant correlation was obtained between the three parameters in the group of PMDs and oral cancer. Thus, concluding that three parameters (serum copper, iron and CIC) show variations independent of each other.

\section{Conclusion}

The present study was designed with the aim of exploring biomarkers for screening and diagnosis of PMDs and OSCC. It was noticed that a definite elevation of CIC and microelement like copper is seen in PMD and OSCC along with a concomitant decline in serum iron levels. However, direct positive correlation between the levels of these parameters and the disease per se was not established.

On the basis of data obtained it can be stated that trace elements and immune complexes do have a close association with the pathogenesis and course of the disease. It can be proposed that biochemical and immumological evaluation and monitoring serves as an important indicator of the disease process thus, assisting screening and early diagnosis. Previous studies concerning serum levels of trace elements and immune complexes have also achieved similar results with varying analytical methods. Automated analyzer used in our study is easily available and has specific filters for trace elements which prove to be an economical as well reliable method for estimation. Further research with larger study groups can be conducted to monitor these parameters in pre-treatment and post-treatment stages of PMDs and oral cancer and assess their role as biomarkers in the progression of the disease. Lastly, it is the concerted efforts and proactive interventions which eventually aid in early detection, management and prognosis of PMDs and oral cancer. 


\section{References}

1. Neville B. Day TA. Oral cancer and precancerous lesions. CA Cancer J Clin. 2002;52(4):195-215. doi:10.3322/canjclin.52.4.195

2. Elango KJ, Anandkrishnan N, Suresh A, Iyer SK, Ramaiyer SK, Kuriakose MA. Mouth self-examination to improve oral cancer awareness and early detection in a high-risk population. Oral Oncol. 2011;47(7):620-4. doi:10.1016/j.oraloncology.2011.05.001

3. Mishra A, Meherotra R. Head and neck cancer: global burden and regional trends in India. Asian Pac J Cancer Prev. 2014;15(2):537-50. doi:10.7314/APJCP.2014.15.2.537

4. Richie JP, Kleinman W, Marina P, Abraham P, Wynder EL, Muscat JE. Blood iron, glutathione, and micronutrient levels and the risk of oral cancer. Nutr Cancer. 2008;60(4):474-82. doi:10.1080/01635580801956477

5. Ayinampudi BK, Narsimhan M. Salivary copper and zinc levels in oral pre-malignant and malignant lesions. J Oral Maxillofac Pathol. 2012;16(2):178-82. doi:10.4103/0973-029X.98452

6. Khanna S, Karjodkar FR. Circulating immune complexes and trace elements (copper, iron and selenium) as markers in oral precancer and cancer: a randomized, controlled clinical trial. Head Face Med. 2006;33(2):1-10. doi:10.1186/1746-160X-2-33

7. Blecher E. Global cancer facts and figures. 2nd ed. Atlanta: American Cancer Society; 2011.

8. Warren S, Gates O. Multiple primary malignant tumors: a survey of the literature and a statistical study. Am J Cancer. 1932;16:1358-414.

9. Tsao AS, Kim ES, Hong WK. Chemoprevention of cancer. CA Cancer J Clin. 2004;54(3):150-80. doi:10.3322/canjclin.54.3.150

10. Slaughter DP, Southwick HW, Smejkal W. Field cancerization in oral stratified squamous epithelium; clinical implications of multicentric origin. Cancer. 1953;6(5):963-8. doi:10.1002/1097-0142(195309)6:5<963:: AID-CNCR2820060515>3.0.CO;2-Q

11. Pai SI, Westra WH. Molecular pathology of head and neck cancer: implications for diagnosis, prognosis and treatment. Annu Rev Pathol. 2009;4(1):49-70. doi:10.1146/annurev.pathol.4.110807.092158

12. Rajendran R, Sellappa S. Serum copper and iron levels in oral squamous cell carcinoma patients: a south Indian study. Adv Appl Sci Res. 2013;4(4):203-6.

13. Saranath D, Mukhopadhyaya R, Rao RS, Fakih AR, Naik SL, Gangal SG. Cell-mediated immune status in patients with squamous cell carcinoma of the oral cavity. Cancer. 1985;56(5):1062-70. doi:10.1002/1097-0142(19850901)56:5<1062:: AID-CNCR2820560517>3.0.CO;2-6

14. Coelho KR. Challenges of the oral cancer burden in India. J Can Epi. 2012;2012:701932. doi:10.1155/2012/701932
15. Llewllyn CD, Johnson NW, Warnakulasuriya KA. Risk factors for squamous cell carcinoma of the oral cavity in young people: a comprehensive literature review. Oral Oncol. 2001;37(5):401-18. doi:10.1016/S1368-8375(00)00135-4

16. Madarić A, Ginter E, Kadrabová J. Serum copper, zinc and copper/zinc ratio in males: influence of aging. Physiol Res. 1994;43(2):107-11.

17. Fairweather-Tait S, Wawer AA, Gillings R, Jennings A, Myint P. Iron status in the elderly. Mech Ageing Dev. 2104;136-7(100):22-8. doi:10.1016/j.mad.2013.11.005

18. Rajendran R. Oral submucous fibrosis: etiology, pathogenesis, and future research. Bull World Health Organ. 1994;72(6):985-96.

19. Balpande AR. Estimation and comparative evaluation of serum iron, copper, zinc and copper/zinc ratio in Oral leukoplakia, submucous fibrosis and oral squamous cell carcinoma. J Indian Acad Oral Med Radiol. 2010;22(2):73-6. doi:10.5005/jp-journals-10011-1018

20. Tokoyuni S. Iron-induced carcinogenesis: the role of redox regulation. Free Radic Bio Med. 1996;20(4):553-66. doi:10.1016/0891-5849(95)02111-6

21. Bhattathiri VN. Paradoxes in iron indices in oral cancer patients vis-a-vis tobacco-alcohol habits. Health Admin. 2006;17(1):76-82.

22. Shetty SR, Babu S, Kumari S, Shetty P, Vijay R, Karikal A. Role of serum trace elements in oral precancer and oral cancer: a biochemical study. J Can Res Treat. 2013;1(1):1-3. doi:10.12691/jcrt-1-1-1

23. Sethuraman R. Estimation of serum iron and serum copper in oral precancer, cancer, and healthy individuals: a comparative study. Eur J Cancer. 2014;50(Suppl 4):e1-11. doi:10.1016/j.ejca.2014.03.051

24. Rajalalitha P. Vali S. Molecular pathogenesis of oral submucous fibrosis: a collagen metabolic disorder. J Oral Pathol Med. 2005;34(6):321-8. doi:10.1111/j.1600-0714.2005.00325.x

25. Karthik H. Nair P. Gharote HP et al. Role of hemoglobin and serum iron in oral submucous fibrosis: a clinical study. ScientificWorldJournal. 2012;2012:254013 doi:10.1100/2012/254013

26. Chester KA, Begent HKJ. Circulating immune complexes (CIC), carcinoembryonic antigen (CEA) and CIC containing CEA as markers for colorectal cancer. Clin Exp Immunol. 1984;58(3):685-93.

27. Saranath D, Mukhopadhyaya R, Rao RS, Fakih AR, Naik SL, Gangal SG. Cell-mediated immune status in patients with squamous cell carcinoma of the oral cavity. Cancer. 1985;56(5):1062-70. doi:10.1002/1097-0142(19850901)56:5<106 2::AID-CNCR2820560517>3.0.CO;2-6

28. Parveen S, Taneja N, Bathi RJ, Deka AC. Evaluation of circulating immune complexes and serum immunoglobulins in oral cancer patients: a follow up study. Indian J Dent Res. 2010;21(1):10-5. doi:10.4103/0970-9290.62800 
29. Rai S, Mody RN. Serum circulating immune complexes as prognostic indicators in premalignant and malignant lesions of oral cavity during and following radiotherapy. J Can Res Ther. 2012;8 Suppl 1:S116-22. doi:10.4103/0973-1482.92225
30. Jane C, Nerurkar AV, Karjodkar FR. Circulating Immune complexes (CIC) as marker for disease progress in oral cancer. Indian J Clin. Biochem. 2007;22(2):114-7. doi: 10.1007/BF02913327 